\title{
Predictive Control Strategies in Power Converters
}

\author{
Ricardo Enrique Pérez-Guzmán, Marco Rivera, and Patrick W. Wheeler
}

\begin{abstract}
Model-based predictive control (MPC) is an attractive solution for controlling power converters. In this article the most recent investigations related to predictive control are addressed and the solutions proposed by the researchers are shown, illustrating also the effectiveness and flexibility of this control technique. In addition, the classification of the most important types of predictive control is introduced and each of them is explained, including some application examples. Predictive control has several advantages that make it suitable for the control of power converters and drives.
\end{abstract}

Keywords-Power converters, model predictive control, power electronics.

\section{INTRODUCCIÓN}

Los convertidores de potencia y sus técnicas de control, han permanecido durante los últimos años en constante desarrollo. $\mathrm{Su}$ aplicabilidad en casi cualquier esfera del desarrollo social como la energía, las comunicaciones, la medicina, la minería o el transporte, impulsan el desarrollo de nuevas investigaciones relacionadas al tema. En particular, las técnicas de control de convertidores han sido un tema de investigación muy activo en el campo de la electrónica de potencia, que cubre innumerables topologías para aplicaciones de baja, media y alta potencia [1]-[5].

Las estrategias de control en convertidores de potencia se pueden clasificar en dos grupos fundamentales: las estrategias lineales y las estrategias no lineales. Dentro de las estrategias lineales destacan los controladores proporcionales $(\mathrm{P})$, proporcionales integrales (PI) y proporcionales integrales derivativos (PID), además de los controladores lineales cuadráticos (LQ/LQR). Su característica fundamental es que se basan en una, o varias etapas de modulación. Por otra parte, las estrategias no lineales se caracterizan por la no linealidad de ciertas variables, cuya magnitud es necesario conocer. Algunos de los ejemplos más importantes son: el control basados en comparadores de histéresis, las técnicas de inteligencia artificial (IA) y el control predictivo. Las técnicas de control más utilizadas en convertidores de potencia se presentan en la Fig. 1.

En los últimos años, el desarrollo de microprocesadores más potentes y el aumento de su capacidad de procesamiento han permitido la evolución de estrategias de control muy eficientes, entre las que se destaca el control predictivo basado en modelo.

El control predictivo basado en modelos (MPC por sus siglas en inglés) es uno de estos métodos, cuyo objetivo

Ricardo Enrique Pérez-Guzmán, (Doctorado en Sistemas de Ingeniería), Departamento de Ciencias de la Computación, Facultad de Ingeniería, Universidad de Talca, Curicó, Chile (e-mail: riperez@utalca.cl).

Marco Rivera, Centro Tecnológico de Conversión de Energías, Facultad de Ingeniería, Universidad de Talca, Curicó, Chile (e-mail: marcoriv@utalca.cl).

Patrick W. Wheeler, Faculty of Science and Engineering, Department of Electrical and Electronic Engineering, University of Nottingham, Nottingham NG7 2RD U.K. (e-mail: pat.wheeler@nottingham.ac.uk).

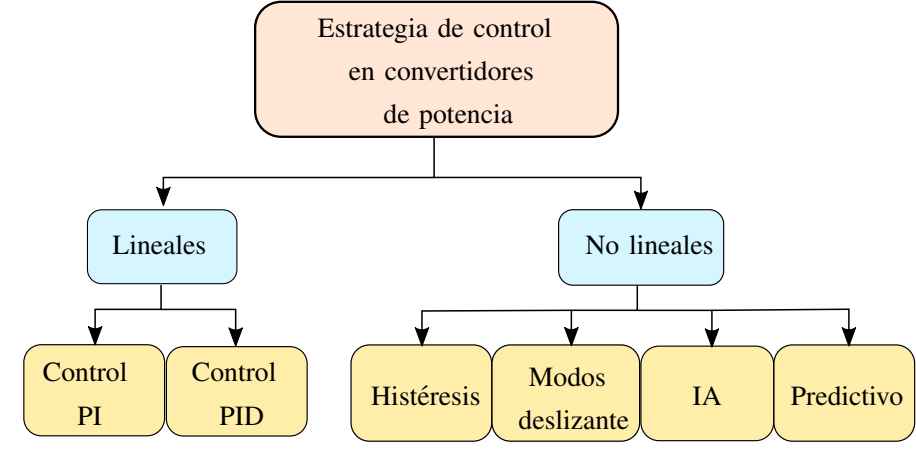

Figura 1. Formas de control para convertidores de potencia.

fundamental es el uso de modelos matemáticos para predecir el comportamiento futuro del sistema y seleccionar acciones de control apropiadas. La inclusión de varios objetivos de control, restricciones y no linealidades en una sola función de costo es la ventaja fundamental de esta popular estrategia de control aplicada en convertidores de potencia. De esta manera es posible controlar algunas variables típicas en convertidores como corriente, voltaje, potencias, torque, o flujo, a través de una función de optimización. Esto se logra mediante la introducción de los objetivos de control en la función de costo.

De manera general, el control predictivo es una técnica de control muy flexible que permite considerar tanto sistemas lineales como no lineales. La ley de control responde a un criterio de optimización y en la misma se pueden incluir más de una variable para predecir el estado deseado del sistema. Sin embargo, esta no es una tarea sencilla, ya que se requiere un modelo dinámico del sistema suficientemente preciso, un algoritmo de optimización, el cual tiene un alto costo computacional. Cada término adicional tiene un factor de peso específico, que se utiliza para manejar la importancia relativa de ese término en relación con el resto de los objetivos. Estos parámetros deben ser diseñados correctamente con el fin de lograr el rendimiento deseado. Lamentablemente, no existen métodos analíticos, numéricos, o teorías de control, para ajustar estos parámetros, y en la actualidad se determinan en base a procedimientos heurísticos [6].

En [7] se muestra el desarrollo de las estrategias de control predictivo en convertidores de potencia. Sin embargo, la variabilidad de posibilidades de control, la aplicación técnicas de optimización más eficientes y el incremento de las necesidades de generación, obligan a los investigadores encontrar nuevas estrategias de control predictivo para aumentar la eficiencia de los sistemas electrónicos de potencia.

En esta investigación se resumen algunos de los retos y tendencias actuales del control predictivo y posibles alternativas de solución. La principal contribución es mostrar una visión ampliada de las principales estrategias de control 


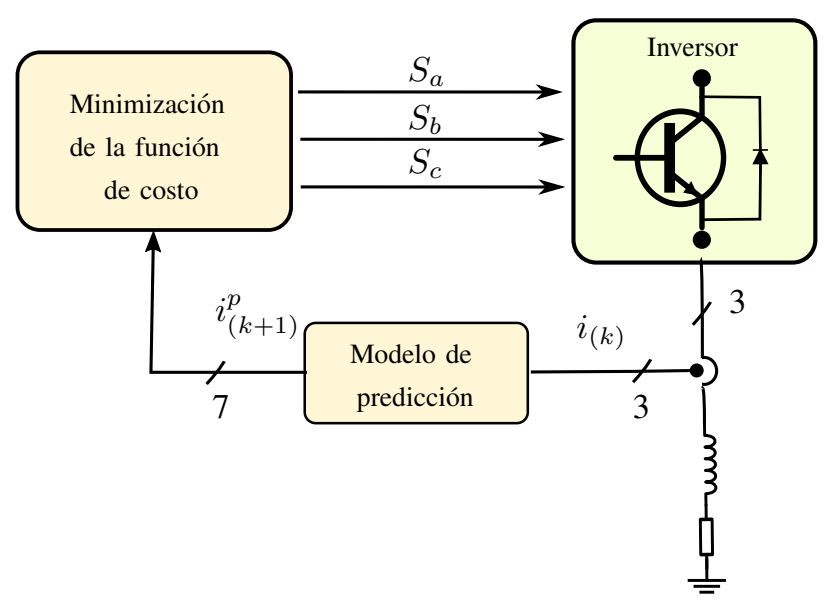

Figura 2. Diagrama en bloques de control predictivo para una carga trifásica

predictivo, algunas de las investigaciones más recientes, así como la propuesta de ventajas y desventajas de cada una de las estrategias. Además, esta investigación sirve de base a los investigadores que inician sus contribuciones en el tema de control predictivo.

\section{CONTROL PREDICTIVO BASAdO EN MODELOS: ESTRATEGIA DE CONTROL}

La estrategia de control predictivo basado en modelos (MPC) se ha utilizado con éxito durante varios años en algunas ramas de la industria. Sin embargo, MPC se ha convertido en una estrategia emergente de control para máquinas eléctricas y convertidores de potencia. En general, hay tres componentes clave en el control predictivo basado en modelo: 1) el modelo de predicción, 2) la función de costo, 3) los algoritmos de optimización [8].

En un convertidor de potencia controlado por MPC sólo se podrán generar un número finito de estados de conmutación y los modelos del sistema se utilizan para predecir el comportamiento de las variables, en cada estado de conmutación [8]. Para la selección del estado de conmutación apropiado que se aplicará, se debe definir un criterio de selección. Este criterio de selección se expresa como una función de costo que será evaluada para los valores de las variables controladas. La predicción del valor futuro de estas variables se calcula para cada posible estado de conmutación. De esta manera, se selecciona el estado de conmutación que minimiza la función de costo [9]. En la Fig. 2 se muestra el modelo clásico de la estrategia de control predictivo aplicada a convertidores de potencia.

Una función de costo puede ser compleja dependiendo de qué variables u objetivos de control se consideran. Sin embargo, estas variables dependen solo de la aplicación en la que estas intervienen. En [9] se recogen algunas funciones de costo que se encuentran en la literatura para aplicaciones de electrónica de potencia. Entre ellos, destacan las funciones que consideran la corriente, el voltaje, el torque o la potencia. Otros objetivos, como la minimización del rizado de tensión, la velocidad y potencia, pueden lograrse incluyendo variables específicas en la función de costo.

La función de costo puede tener cualquier forma, pero en general, se puede escribir como:

$$
g=\sum_{\ell=k+1}^{k=N_{\rho}} \tilde{x}_{\ell}^{T} Q \tilde{x_{\ell}}+\sum_{\gamma=k}^{k+N_{c}-1} \mathbf{u}_{\gamma}^{T} R \mathbf{u}_{\gamma}
$$

donde $\tilde{x}_{\ell}=\tilde{x}_{\ell}-\tilde{x}_{\ell^{*}}$ es un vector en el que cada componente representa la diferencia entre los valores estimados $\tilde{x}_{j, \ell}$ y la referencia, $\tilde{x}_{j, \ell}^{*}$, para cualquier variable $x_{j}$ en el instante $\ell$. Por su parte, $u_{\gamma}$ es un vector de entradas de control $u_{i}$ en el instante $\gamma$, y $N_{p}$ y $N_{c}$ son los horizontes de predicción y control, respectivamente [9].

Cuando $Q$ y $R$ son diagonales, entonces la ecuación anterior se puede expresar como [9]:

$$
g=\sum_{\ell=k+1}^{k+N_{\rho}} \sum_{\jmath=0}^{m-1} \lambda_{j}\left(\hat{x}_{j, \ell}-x_{\jmath, \ell}^{*}\right)^{2}+\sum_{\gamma=k}^{k+N_{c}-1} \sum_{\imath=0}^{n-1} \lambda_{\imath}\left(u_{\imath, \gamma}\right)^{2}
$$

donde $\lambda_{\jmath}$ y $\lambda_{i}$ son los factores de ponderación asociados a la variable $x_{j}$ y la acción de control $u_{i}$, respectivamente.

Como se puede apreciar, la complejidad de una correcta selección de la función de costo aumenta, con el aumento de los objetivos de control, lo que implica que la disminución de error de la predicción sea un elemento muy importante a considerar. Algunos investigadores han demostrado que este error puede ser calculado como la ecuación (2), cuando los objetivos de control consideran una variable objetivo [9]. En cambio, si presenta dos, o más términos los mejores resultados los ofrece la función de costo cuadrada como se muestra en la ecuación (3) [9]. El error cuadrado presenta mejor seguimiento de la referencia cuando se agregan términos adicionales a la función de costo. La función de costo considera la trayectoria de las variables entre los intervalos de tiempo $t_{k}$ y $\left.t_{(} k+1\right)$ conduciendo a la minimización del error medio, lo que implica un seguimiento más preciso. Además, la selección de la función de costo correcta es más difícil cuando se incluyen varios objetivos de control en el problema de optimización.

$$
\begin{gathered}
g=\left|x^{*}-x^{p}\right| \\
g=\left(x^{*}-x^{p}\right)^{2}
\end{gathered}
$$

Una de las principales ventajas de MPC, es que la función de costo admite cualquier término necesario que pueda representar una predicción para otra variable del sistema, así como las restricciones del mismo. Dado que estos términos pueden ser de una naturaleza física diferente (tensión, potencia reactiva, pérdidas de conmutación, torque, flujo, entre otros) sus unidades y magnitudes también puede ser diferentes. La solución a este problema ha sido abordada de diferentes maneras, aunque algunas de ellas coinciden en incluir coeficientes de peso, o factores de ponderación $\lambda$, para cada término de la función de costo como se muestra en la ecuación (4).

$$
g=\lambda_{x}\left\|x^{*}-x^{p}\right\|+\lambda_{y}\left\|y^{*}-y^{p}\right\| \ldots+\lambda_{z}\left\|z^{*}-z^{p}\right\|
$$

Según el criterio de algunos investigadores [5], [8], [9], 
las alternativas de control predictivo más utilizadas en convertidores de potencia son: MPC de estado continuo y MPC de estado finito. Dentro de esta última se encuentran otras formas de control: MPC modulado, y MPC multi-objetivo. A continuación se describen los últimos avances en cada una de estas clasificaciones.

\section{PRinCiPAles EStrategias De CONTROL MPC}

\section{A. MPC de estado continuo (CCS-MPC)}

La estrategia de control CCS-MPC asume una naturaleza continua del convertidor, es decir, no se toman en cuenta los estados de conmutación de los semiconductores dentro del algoritmo de control. Por lo tanto, se genera una señal de control continua. De esta manera, para generar los estados de conmutación se utiliza un modulador (PWM o SVPWM) [10], lo que produce una salida con una frecuencia fija. La característica continua de este tipo de modulación es lo que le otorga el nombre a esta estrategia de control.

Uno de los retos más importantes que afecta el desempeño de cualquier modelo MPC es el incremento del contenido armónico [2]. CCS-MPC presenta algunas ventajas en comparación a otras formas de control, debido a la utilización de un modulador de frecuencia fija, lo que implica una respuesta dinámica más rápida y menor contenido armónico. Además, se pueden utilizar horizontes de predicción mayores, sin la necesidad de incrementar significativamente el costo computacional.

En cambio, la utilización de un modelo lineal limita la aplicación del mismo a determinados puntos de operación. Por este motivo, si se requiere una modelación no lineal, se deberán crear modelos lineales para distintos puntos de operación. Lo anterior implica que la complejidad a la hora de formular el modelo sea alta y se necesite un modulador. Para resolver estos inconvenientes se desarrollan estrategias de control como MPC de estado finito.

\section{B. MPC de estado finito (FCS-MPC)}

La necesidad de encontrar una solución a la complejidad del modelo y la utilización de moduladores, son los principales inconvenientes de CCS-MPC. Para resolver estos inconvenientes FCS-MPC toma en cuenta la naturaleza discreta de los convertidores para formular un algoritmo menos complejo, que no requiere modulación. De esta manera, el estado que minimice la función de costo será seleccionado [11]. Al tener un número finito de posibles estados y acciones de control, este enfoque se denomina MPC de estado finito (FCS-MPC, por su nombre en inglés Finite Control Set MPC).

El modelo FCS-MPC es una de las alternativas más atractivas en la actualidad debido a su rápida respuesta dinámica, la fácil inclusión de no linealidades y restricciones dentro del modelo de predicción, su simpleza y la ausencia de moduladores o lazos de corriente dentro de la estrategia de control. En cambio, su desarrollo estuvo limitado por la alta capacidad computacional que requiere este modelo. Incluso para pequeños sistemas eléctricos, se necesita una elevada frecuencia de computación del algoritmo de control predictivo para disminuir el contenido armónico (THD). La evolución de

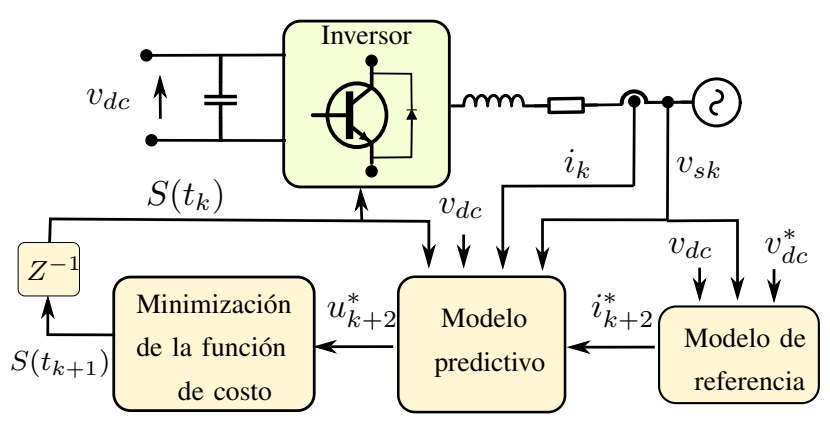

Figura 3. Esquema de control FCS-MPC.

dispositivos programables como FPGA, con procesadores más potentes, ha permitido solucionar algunos de estos inconvenientes.

En la Fig. 3 se muestra el esquema de control de este algoritmo. Las aplicaciones de esta técnica de control son diversas y varían desde inversores de dos niveles trifásicos [12], [13], inversores de tres niveles (NPC) [14], convertidores matriciales [15], entre otros. En cada una de las topologías anteriores se tiene una cantidad limitada de estados para aplicar MPC.

\section{MPC modulado $\left(M^{2} P C\right)$}

Debido a la necesidad de mitigar las insuficiencias de FCSMPC, referidas al incremento computacional y el incremento del rizado en las variables de control del algoritmo [16], algunas investigaciones proponen modificar los estados de conmutación de FCS-MPC, para mejorar la secuencia de control [17]. Por otro lado, la utilización de modulación de espacios vectoriales (SVM), reduce el rizado de las variables de control y permite incrementar el horizonte de predicciones [18].

De esta manera, se propone en la literatura MPC modulado $\left(M^{2} P C\right)$ y cuyo objetivo es incluir un esquema de modulación dentro de la función de costo del algoritmo MPC [19]. Cada estado de conmutación se calcula a partir de SVM, lo que implica reducir los armónicos a la salida del convertidor producto de la etapa de modulación previa. Además, los estados de salida son calculados a una frecuencia fija.

En la Fig. 4 se describe el esquema de control de esta arquitectura. El bloque MPC modulado $\left(M^{2} P C\right)$ define una secuencia de dos vectores de tensión $S 1$ y $S 2$ y dos valores, $G 1$ y $G 2$, proporcionales a sus tiempos de aplicación. Una segunda etapa calcula los tiempos de aplicación finales utilizando la información del bloque $M^{2} P C$.

\section{MPC multi-objetivo (MO-MPC)}

El principal problema de las estrategias de control descritas anteriormente es el ajuste de los parámetros del controlador (selección adecuada de los pesos dentro de la función de costo) [6]. Una solución interesante resulta encontrar mecanismos que no incluyan el ajuste de estos parámetros. Tal es el caso de la formulación multi-objetivo, cuyo rol fundamental es minimizar la función de costos evitando el ajuste de los factores de pesos. 
El concepto de optimización multi-objetivo aplicado a convertidores de potencia es un concepto relativamente nuevo. En [20], se propone una estrategia en cascada de controladores con lógica difusa FCS-MPC. En un primer paso se propone la estrategia para reducir la frecuencia de conmutación y un controlador difuso para escoger los factores de peso de manera dinámica. Por otra parte, en [21], se presenta una estrategia de control para los generadores de inducción doblemente alimentados (DFIGs) basados en el esquema de control predictivo del modelo multi-objetivo (MO-MPC). El comportamiento futuro del DFIG se predice utilizando el modelo del sistema y los posibles estados de conmutación del convertidor. Finalmente, en [22], se utiliza un modelo de control predictivo de estados finitos (FS-MPC) basado en lógica difusa, para mejorar la estabilidad y los cambios durante el estado estacionario del convertidor. Esta propuesta tiene dentro de sus objetivos evitar los problemas relacionados con los ajustes de peso sin afectar el rendimiento de la estrategia de control.

Un elemento importante de esta estrategia de control es que se basa en los mismos principios de funcionamiento de FCSMPC. Ello implica que las desventajas con respecto al horizonte de predicción encontradas en FCS-MPC, se encuentran presentes en MO-MPC. Incluso, el algoritmo de optimización añade complejidad computacional, por lo que esta estrategia requerirá mayores recursos computacionales. Da ahí que algunos autores consideren prácticamente imposible implementarla en inversores multiniveles [6]. En la Tabla I se muestran algunos de los elementos más importantes de las estrategias de control abordadas.

\section{Discusión}

Las estrategias de control MPC son una de las alternativas de control más utilizadas en electrónica de potencia y máquinas eléctricas en los últimos años. Sin embargo, todavía es necesario profundizar en algunos temas como la elección de los factores de peso, las estrategias inteligentes de control, ampliar el horizonte de predicción, o el rendimiento computacional del controlador. Por ejemplo, para garantizar la estabilidad de un inversor de voltaje (VSI), es necesario ajustar los factores de peso considerando incluso posibles cambios en la carga.

Se pudo comprobar en la literatura que dentro de las estrategias MO-MPC destacan los algoritmos de lógica difusa, por los bajos requisitos computacionales que utiliza y los buenos

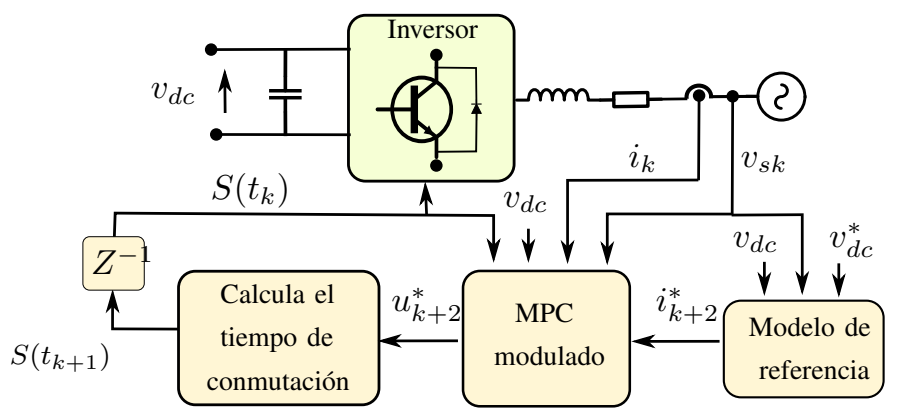

Figura 4. Esquema de control $M^{2} P C$ resultados sobre todo en la selección de los factores de peso. No obstante, algoritmos de optimización como FCS-MPC simplificado, FCS-MPC multipasos, o FSC-MPC jerárquico también son alternativas recientes de control, que reducen el costo computacional y son descritas en [9].

La función de costo otorga flexibilidad al sistema de control y permite optimizar parámetros como la potencia, la frecuencia de conmutación, el torque o el control de un motor. Su mayor inconveniente es que requiere un modelo matemático apropiado y conocido previamente. Varios investigadores coinciden en que existen tres elementos importantes a considerar cuando se aplica MPC y son: i) disminución del costo computacional y con ello el horizonte futuro de predicción [23], ii) el ajuste de los factores de peso [24] y iii) el incremento de la eficiencia del convertidor [2].

Alternativas para mejorar el rendimiento y la calidad del sistema en estado estacionario se ha propuesto utilizar una técnica de control con un esquema de modulación $\left(M^{2} P C\right)$. Sin embargo, en la función de costo también se requiere de un factor de ponderación, por lo que se mantiene la complejidad. Por otro lado, el desempeño de MPC frente a un horizonte de predicción amplio en estado estacionario, favorece la eficiencia de la estrategia de control. Su limitación fundamental sigue siendo ajustar el tamaño del horizonte de predicción, lo que incrementa el costo computacional.

A pesar del incremento de la capacidad computacional de los dispositivos en la actualidad, en algunos casos no es posible realizar un control predictivo adecuado, debido a la falta de procesamiento. Un ejemplo de esto resultan los convertidores multi-fase y multi-nivel, donde en cada tiempo de muestreo tienen que evaluar el modelo de predicción para una gran cantidad de estados de conmutación.

Por último, se ha evidenciado la factibilidad, e interés de la comunidad en las estrategias de control predictivo aplicado a convertidores de potencia. No obstante, aún quedan elementos por abordar en investigaciones futuras.

\section{TRABAJOS FUTUROS}

El control predictivo en convertidores de potencia es una potencial rama de investigación en la actualidad. Elementos como la reducción del error de seguimiento de las referencias, el desempeño del convertidor en estado estacionario, o la optimización de las variables consideradas, son algunos de los elementos que se deben considerar como áreas incipientes de investigación. A continuación se relacionan los temas potenciales para la investigación futura:

- Para mejorar la respuesta en estado estacionario, es posible definir un horizonte de predicción extendido, combinado con una reducción de la frecuencia de conmutación, pueden ser utilizados para disminuir el THD [25].

- Se necesitan nuevos aportes referidos a la estabilidad y selección óptima del factor de peso. No existe, hasta el momento, ninguna forma establecida de demostrar la estabilidad del control predictivo y seleccionar de manera óptima, los factores de ponderación [9].

- Varios algoritmos MPC se centran en la aplicación de métodos de frecuencia fija para mejorar el contenido 
Tabla I

COMPARACIÓN DE LOS DISTINTOS ENFOQUES DE CONTROL EN CONVERTIDORES DE POTENCIA, BASADA EN [6].

\begin{tabular}{|c|c|}
\hline Estrategia de control & Ventajas \\
\hline Control lineal & $\begin{array}{l}\text { Se conoce el ancho de banda. } \\
\text { Fácil de extender a otras topologías. } \\
\text { Frecuencia de conmutación fija. } \\
\text { Muy utilizado en aplicaciones comerciales. }\end{array}$ \\
\hline $\begin{array}{l}\text { Control basado } \\
\text { en histéresis }\end{array}$ & $\begin{array}{l}\text { No es necesario un modulador. } \\
\text { Buen desempeño dinámico del sistema. } \\
\text { Control no lineal robusto. } \\
\text { Su diseño es relativamente simple. } \\
\text { Es utilizado en aplicaciones comerciales. }\end{array}$ \\
\hline $\begin{array}{l}\text { MPC de estado } \\
\text { continuo } \\
\text { (CCS-MPC) }\end{array}$ & $\begin{array}{l}\text { Frecuencia de conmutación fija. } \\
\text { El comportamiento dinámico del sistema } \\
\text { se logra predecir de manera correcta. } \\
\text { Varios objetivos en una sola ley de control. } \\
\text { Extiende el horizonte de predicción sin aumentar } \\
\text { el tiempo de procesamiento. } \\
\text { El contenido armónico se reduce debido a } \\
\text { la etapa de modulación. }\end{array}$ \\
\hline
\end{tabular}

Desventajas

Se requiere un modulador (PWM, SVPWM).

Falta de estabilidad con sistemas no lineales.

Cambios en las restricciones del modelo no pueden incluirse.

Se requiere una transformación de coordenadas.

La frecuencia de muestreo debe ser alta.

Presenta problemas de resonancia.

Complejidad para extenderlo a otras topologías.

Frecuencia de conmutación variable.

La selección errónea de los elementos del controlador puede empeorar.

Se requiere un modulador (PWM, o SVPWM). Adaptarse a los cambios en la dinámica del sistema no es una tarea sencilla.

Dificultad al incluir restricciones en la ley de control.

Se puede ajustar la frecuencia de conmutación.

Baja complejidad sin necesidad de modulador.

Rizado importante a la salida del convertidor.

Múltiples objetivos a la vez con una

El rizado se disminuye aplicando estrategias

MPC de estado

sola ley de control.

computacionales más complejas.

Las restricciones del sistema se incluyen en la

Extender el horizonte de predicción aumenta

(FCS-MPC)

ley de control.

de forma considerable el tiempo de cálculo.

Estrategias de control muy utilizada en converti-

dores de potencia y aplicaciones industriales.

La selección de los parámetros del controlador

deben ajustarse de manera correcta

para permitir el rendimiento del sistema.

Las aplicaciones prácticas requieren mayor esfuerzo e investigación.

Las ventajas de MPC se mantienen en esta estrategia.

MPC Modulado

Posee un buen perfil de armónicos dada la

Al aumentar el horizonte de predicción, etapa de modulación.

el tiempo computacional aumentará considerablemente.

No se requiere un modulador, ni ajustar los

parámetros del controlador.

MPC multiobjetivo

La función objetivo, maneja las restricciones

de manera sencilla.

Se requiere un alto costo computacional

(MO-MPC)

La respuesta dinámica es muy rápida.

armónico de las señales controladas [26], [27]. Esto conduce a nuevas posibilidades en las estrategias de control, manteniendo la ventaja de no usar un modulador, pero aplicando el estado de conmutación óptimo durante un período específico.

- Existen insuficiencias en la validación de los algoritmos de control propuestos en aplicaciones específicas, que generalmente requieren un alto rendimiento computacional, como la generación de energía o vehículos eléctricos.

\section{CONCLUSIONES}

El control predictivo basado en modelo (MPC) es una solución muy atractiva para controlar las aplicaciones en electrónicas de potencia. Se abordó el principio de funcionamiento de MPC, concluyendo que la implementación de MPC depende de tres elementos clave, i) el modelo de predicción, ii) la función de costo y iii) el algoritmo de optimización. Varios aspectos relacionados con estos temas han sido investigados en la literatura. Los más relevantes son la selección de la función de costos, el diseño del factor de peso, la reducción del costo computacional y la extensión de los horizontes de predicción.

El método de control FCS-MPC es uno de los más utilizados 
por su simplicidad y el control de diferentes magnitudes sin requerir técnicas de modulación adicionales o bucles internos de control en cascada. No obstante, la estrategia de control predictivo más adecuada dependerá de la aplicación y de los requisitos del sistema.

\section{AgRAdECIMIENTOS}

Los autores desean agradecer al Programa FONDECYT Regular a través del proyecto 1160690 y a la Beca de Doctorado Nacional CONICYT 2019, folio 21190709.

\section{REFERENCIAS}

[1] F. Gavilán, S. Toledo, M. Rivera, D. Caballero, E. Maqueda, and R. Gregor, "Predictive current control strategy for a direct matrix converter with modulated switching pattern," in 2018 IEEE International Conference on Automation/XXIII Congress of the Chilean Association of Automatic Control (ICA-ACCA). IEEE, 2018, pp. 1-6.

[2] M. Rivera, S. Toledo, C. Baier, L. Tarisciotti, P. Wheeler, and S. Verne, "Indirect predictive control techniques for a matrix converter operating at fixed switching frequency," in 2017 IEEE International Symposium on Predictive Control of Electrical Drives and Power Electronics (PRECEDE). IEEE, 2017, pp. 13-18.

[3] Z. Zhang, H. Fang, F. Gao, J. Rodríguez, and R. Kennel, "Multiplevector model predictive power control for grid-tied wind turbine system with enhanced steady-state control performance," IEEE Transactions on Industrial Electronics, vol. 64, no. 8, pp. 6287-6298, 2017.

[4] F. An, W. Song, B. Yu, and K. Yang, "Model predictive control with power self-balancing of the output parallel dab dc-dc converters in power electronic traction transformer," IEEE Journal of Emerging and Selected Topics in Power Electronics, vol. 6, no. 4, pp. 1806-1818, 2018.

[5] P. Acuna, R. P. Aguilera, A. M. Ghias, M. Rivera, C. R. Baier, and V. G. Agelidis, "Cascade-free model predictive control for single-phase grid-connected power converters," IEEE Transactions on Industrial Electronics, vol. 64, no. 1, pp. 285-294, 2017.

[6] P. Trigo, "Diseño de estrategias de control predictivo multi-objetivo para un filtro activo en paralelo trifásico de tres hilos," Ph.D. dissertation, Tesis Doctoral, Universidad de Chile, 2016.

[7] M. Sarbanzadeh, M. A. Hosseinzadeh, E. Sarebanzadeh, and M. Rivera, "Trends and challenges of predictive control in power electronics," in 2018 IEEE International Conference on Automation/XXIII Congress of the Chilean Association of Automatic Control (ICA-ACCA). IEEE, 2018, pp. 1-6.

[8] F. Wang, X. Mei, J. Rodriguez, and R. Kennel, "Model predictive control for electrical drive systems-an overview," CES Transactions on Electrical Machines and Systems, vol. 1, no. 3, pp. 219-230, 2017.

[9] S. Vazquez, J. Rodriguez, M. Rivera, L. G. Franquelo, and M. Norambuena, "Model predictive control for power converters and drives: Advances and trends," IEEE Transactions on Industrial Electronics, vol. 64, no. 2, pp. 935-947, 2017.

[10] X. Yue, X. Wang, and F. Blaabjerg, "Review of small-signal modeling methods including frequency-coupling dynamics of power converters," IEEE Transactions on Power Electronics, vol. 34, no. 4, pp. 3313-3328, 2018.

[11] F. Wang, X. Mei, J. Rodriguez, and R. Kennel, "Model predictive control for electrical drive systems-an overview," CES Transactions on Electrical Machines and Systems, vol. 1, no. 3, pp. 219-230, 2017.
[12] H. T. Nguyen, E.-K. Kim, I.-P. Kim, H. H. Choi, and J.-W. Jung, "Model predictive control with modulated optimal vector for a threephase inverter with an lc filter," IEEE Transactions on Power Electronics, vol. 33, no. 3, pp. 2690-2703, 2018.

[13] M. Aguirre, S. Kouro, C. A. Rojas, J. Rodriguez, and J. I. Leon, "Switching frequency regulation for fcs-mpc based on a period control approach," IEEE Transactions on Industrial Electronics, vol. 65, no. 7, pp. 5764-5773, 2018

[14] T. Jing and A. S. Maklakov, "A review of voltage source converters for energy applications," in 2018 International Ural Conference on Green Energy (UralCon). IEEE, 2018, pp. 275-281.

[15] K. Sreeram, "Universal matrix converter for ac and dc power conversions," in 2017 IEEE International Conference on Circuits and Systems (ICCS). IEEE, 2017, pp. 392-397.

[16] A. Godlewska, "Finite control set model predictive control of current source inverter for photovoltaic systems," in 2018 14th Selected Issues of Electrical Engineering and Electronics (WZEE). IEEE, 2018, pp. $1-4$.

[17] S. Fuchs and J. Biela, "Impact of the prediction error on the performance of model predictive controllers with long prediction horizons for modular multilevel converters-linear vs. nonlinear system models," in 2018 20th European Conference on Power Electronics and Applications (EPE'18 ECCE Europe). IEEE, 2018, pp. P-1.

[18] L. Cheng, P. Acuna, R. P. Aguilera, J. Jiang, S. Wei, J. E. Fletcher, and D. D. Lu, "Model predictive control for $\mathrm{dc}-\mathrm{dc}$ boost converters with reduced-prediction horizon and constant switching frequency," IEEE Transactions on Power Electronics, vol. 33, no. 10, pp. 9064-9075, 2017.

[19] L. Tarisciotti, P. Zanchetta, A. Watson, S. Bifaretti, and J. C. Clare, "Modulated model predictive control for a seven-level cascaded h-bridge back-to-back converter," IEEE Transactions on industrial electronics, vol. 61 , no. 10 , pp. 5375-5383, 2014.

[20] X. Liu, D. Wang, and Z. Peng, "Cascade-free fuzzy finite-control-set model predictive control for nested neutral point-clamped converters with low switching frequency," IEEE Transactions on Control Systems Technology, no. 99, pp. 1-8, 2018

[21] J. Hu, Y. Li, and J. Zhu, "Multi-objective model predictive control of doubly-fed induction generators for wind energy conversion," IET Generation, Transmission \& Distribution, vol. 13, no. 1, pp. 21-29, 2018.

[22] X. Liu, D. Wang, and Z. Peng, "Multi-objective fuzzy-decision-makingbased fs-mpc with improved performance for grid-connected converters," Electrical Engineering, vol. 100, no. 4, pp. 2439-2456, 2018.

[23] M. Rana, I. Koprinska, and V. G. Agelidis, "Univariate and multivariate methods for very short-term solar photovoltaic power forecasting," Energy Conversion and Management, vol. 121, pp. 380-390, 2016.

[24] R. Razani, M. H. Ravanji, and M. Parniani, "An enhanced hybrid modular multilevel converter for improved performance," IEEE Transactions on Power Electronics, 2018.

[25] T. Geyer, Model predictive control of high power converters and industrial drives. John Wiley \& Sons, 2016.

[26] F. Xiao, L. Dong, L. Li, and X. Liao, "A frequency-fixed sogi-based pll for single-phase grid-connected converters," IEEE Transactions on Power Electronics, vol. 32, no. 3, pp. 1713-1719, 2017.

[27] Y. Yang, K. Zhou, and F. Blaabjerg, "Enhancing the frequency adaptability of periodic current controllers with a fixed sampling rate for gridconnected power converters," IEEE Transactions on Power Electronics, vol. 31, no. 10, pp. 7273-7285, 2016. 